\title{
Use of polyvinylpyrrolidone in the testing of staphylococci for sensitivity to methicillin and cephradine
}

\author{
R. BAYSTON \\ From the University Department of Paediatrics, Children's Hospital, Sheffield S10 2TH, UK
}

SUMMARY The use of polyvinylpyrrolidone, an inert polymer resembling plasma proteins in itsic colligative effects, in the testing of micrococcaceae for sensitivity to methicillin and cephradine is + described. Generally results are quite comparable with those of conventional methods. The absences of any inhibitory effect of the polymer compared to sodium chloride, and its physiological inertia compared to sucrose, along with its suitability for sterilisation by autoclaving are seen as advantages It is suggested that the use of this substance may give results which are more applicable to the in vivo situation. This may apply particularly in the case of cephradine.

It has been shown that when testing Staphylococcus pyogenes for sensitivity to methicillin lower incubation temperatures favour the growth of resistant cocci, with the result that more strains are found to be resistant to the antibiotic when the test is carried out at $30^{\circ} \mathrm{C}$ rather than $37^{\circ} \mathrm{C}$ (Annear, 1968). If the test is carried out at $37^{\circ} \mathrm{C}$, then the addition of sodium chloride to the medium in a final concentration of $5 \%$ also results in a greater number of resistant strains detected (Churcher, 1968). The additional sodium chloride is thought to prevent lysis of the surviving cocci, which are deficient in cell-wall material (Williams, 1963; Pratt, 1966), by increasing the osmotic strength of the medium (Barber, 1964). Because cephalosporins also exert their main effect on the cell-walls of bacteria it was thought that the use of low incubation temperatures and osmotic support may have a similar effect on the rate of detection of resistant staphylococci. On investigation it was found that some strains of micrococcaceae when tested on sodium chloride agar failed to grow on control plates containing no antibiotic, and that some other strains showed only feeble growth. These strains appeared to be inhibited by the sodium chloride, and the reality of the apparent sensitivity of the feeble-growers to the antibiotic in such circumstances was questionable.

Received for publication 6 October 1977

\section{Material and methods}

Except in the disc diffusion tests the basic medium $\stackrel{\mathbb{}}{\mathscr{2}}$ used was nutrient agar (LAB M). To one batch $\vec{\sigma}$ of this were added sodium chloride in a finaB concentration of $5 \%$ and methicillin in a fina concentration of $10 \mu \mathrm{g} / \mathrm{ml}$ (medium A). To second batch were added sodium chloride as above and cephradine in a final concentration of $50 \mu \mathrm{g} / \mathrm{m}$ ? (medium B). A third batch consisted of nutrient agar with PVP added to a final concentration of $4 \%$ and methicillin as above (medium C). Medium DS consisted of nutrient agar with PVP as in medium $\mathrm{C}_{\mathrm{O}}$ but with cephradine added to give a final concentration of $50 \mu \mathrm{g} / \mathrm{ml}$.

The organisms tested consisted of 60 strains of Staph. pyogenes, 24 of which were known to bes resistant to methicillin on routine testing; 40 strains of Staph. albus, many from clinical infections such as ventriculitis or colonised hydrocephalus shunts
and 22 micrococci from various sources.

Four-hour peptone water cultures of the organismse to be tested were spot-inoculated onto plates of thes above media and these were then incubated over:night at $30^{\circ} \mathrm{C}$. Control plates containing nutrient agar and either sodium chloride or PVP but nơ antibiotics were also included.

For disc-diffusion tests four-hour peptone water cultures of the organisms were inoculated onto DST agar (LAB M), and a sensitivity disc containing 434 
$30 \mu \mathrm{g}$ cephradine was applied. These plates were incubated overnight at $37^{\circ} \mathrm{C}$.

\section{Results}

In most cases there was little difference in growth on the medium containing sodium chloride from that on PVP medium.

However, two strains of Staph. albus and three strains of micrococcus grew feebly on sodium chloride agar and appeared to be inhibited by the salt, whereas they grew normally on the PVP medium. When tested on these two media containing either methicillin or cephradine, they again grew well on the PVP medium but did not grow at all on that containing sodium chloride. Thus they appeared to be resistant to both cephradine and methicillin on PVP agar, but sensitive to these two antibiotics on sodium chloride agar.

In general, and in the case of these five organisms in particular, PVP medium was felt to give a more clear-cut picture with very few equivocal results.

When the results of testing organisms for sensitivity to cephradine by the disc diffusion method at $37^{\circ} \mathrm{C}$ were compared with those of the PVP agar method at $30^{\circ} \mathrm{C}$, two strains of Staph. pyogenes, three strains of Staph. albus, and three strains of micrococcus were found to be sensitive on the disc test but resistant on the PVP agar.

\section{Discussion}

An important consequence of the contact between many bacteria and cell-wall-active antibiotics is the formation of cell-wall-deficient filamentous or bulbous forms. Greenwood and O'Grady (1972, 1973 , 1974) have shown that, by altering the osmolality of the culture medium, it is possible to increase the numbers of surviving spheroplasts to a point where the organism is able to re-establish itself. The substance used in one set of their experiments to raise the osmolality was sucrose. Pilot experiments with a small number of strains using this substance in the test media along with either methicillin or cephradine gave results which were similar to those described here, but PVP was chosen for further study rather than sucrose because of the advantages described below.

PVP is widely used in the cosmetic and pharmaceutical industries. It has also found application as a plasma expander in burned patients, and in the prevention of kernicterus in jaundiced babies (Kutor, 1958). It is also a well-known constituent of povidone iodine surgical scrub. Lawson and Douglas (1973) used media containing PVP in order to induce and maintain L-forms of gonococci. Its molecular weight can be made to vary between 1000 and 360000 , depending on the degree of polymerisation, and the lower-molecular-weight forms are watersoluble. Two important advantages which PVP has over sucrose are that it is not metabolised by organisms and that it can be sterilised by autoclaving. It is physiologically inert so far as bacteria are concerned, apart from its protective properties, which resemble those of serum proteins (Jirgensons, 1951).

The results of this investigation show that PVP is superior to sodium chloride in media for testing staphylococci and micrococci for methicillin sensitivity, on the grounds that some strains of coagulase-negative micrococcaceae appear to be inhibited by sodium chloride. The apparent 'sensitivity' of certain strains to methicillin in such circumstances may therefore be a function of the combined actions of sodium chloride and the antibiotic.

When the results on cephradine-PVP media were compared to those of the cephradine disc diffusion method, fewer sensitive strains were found. Some of the discrepancy was undoubtedly due to difficulty in interpretation of the disc test in some cases where zone diameters were equivocal. This was so in the case of the two strains of Staph. pyogenes, which gave different results on the two media. The organisms tested on PVP media with antibiotic either failed to grow at all or grew normally, and interpretation was far easier. However, some strains which were obviously sensitive by the disc test were resistant on PVP medium, and the discrepancy was not due to differences in interpretation alone. The results on PVP medium with cephradine were identical with those with methicillin on that medium.

The differences in results between conventional and PVP media were therefore confined mainly to coagulase-negative micrococcaceae. These organisms are being incriminated more often as the causative agents of disease. In the case of Staph. albus, infections of the central nervous system and a variety of implantable prostheses have been recorded; micrococci have mainly been found as urinary tract pathogens. From the point of view of prospective antibiotic treatment, as well as the fact that these organisms, being 'background flora', are affected by most antibiotics by whatever route they are administered, and therefore may reflect changes in the general picture of antibiotic resistance, it is important to obtain accurate and valid data as to their sensitivities.

\section{Conclusions}

PVP appears to offer the advantages of sodium chloride in methicillin sensitivity testing without 
being inhibitory to less vigorous strains, and its use in the testing of cephradine sensitivity as described gives results which are completely comparable to those obtained with methicillin.

Moreover, because of the similarity in behaviour between serum proteins and PVP, the use of this substance in sensitivity tests may give results which are more relevant to conditions which pertain in vivo.

I am grateful to $\mathrm{Mr} \mathrm{R}$. Gledhill, of the Public Health Laboratory, Northern General Hospital, Sheffield, for some of the strains of Staph. pyogenes, and to the staff of the Bacteriology Department, Children's Hospital, Sheffield, for their co-operation. Thanks are also due to Dr Sheena Waitkens, late of Sheffield University, for interesting discussion.

\section{References}

Annear, D. I. (1968). The effect of temperature on resistance of Staphylococcus aureus to methicillin and some other antibiotics. Medical Journal of Australia, 1, 444-446.

Barber, M. (1964). Naturally-occurring methicillinresistant staphylococci. Journal of General Microbiology, 35, 183-190.

Churcher, G. M. (1968). A screening test for the detection of methicillin-resistant staphylococci. Journal of Clinical Pathology, 21, 213-217.
Greenwood, D., and O'Grady, F. (1972). The effect of osmolality on the response of Escherichia coli and Proteus mirabilis to penicillins. British Journal of Experimental Pathology, 53, 457-464.

Greenwood, D., and O'Grady, F. (1973). Comparison? of the responses of Escherichia coli and Proteus mira-음 bilis to seven beta-lactam antibiotics. Journal of $\frac{\sqrt{\sigma}}{\vec{\sigma}}$ Infectious Diseases, 128, 211-222.

Greenwood, D., and O'Grady, F. (1974). The comparative@ performance of beta-lactam antibiotics against ampicillin-sensitive Escherichia coli in conditions simulating. those of the infected urinary bladder. British Journal $\overrightarrow{\vec{C}}$ of Experimental Pathology, 55, 245-250.

Jirgensons, B. (1951). Viscosity, precipitation and protective effect of PVP. Makromolekuläre Chemie, , 6, 30-38.

Kutor, J. (1958). Prevention of kernicterus in prematurecr infants by Periston (Hungarian). Gyermekgyógyászat, $\underset{\oplus}{\stackrel{\oplus}{\oplus}}$

Lawson, J. W., and Douglas, J. T. (1973). Induction and응 reversion of the L-form of Neisseria gonorrhoeae. Canadian Journal of Microbiology, 19, 1145-1151.

Pratt, B. C. (1966). Cell-wall deficiencies in L-forms of Staphylococcus aureus. Journal of General Microbiology, 42, 115-122.

Williams, R. E. O. (1963). L-forms of Staphylococcus. aureus. Journal of General Microbiology, 33, 325-334.

Requests for reprints to: R. Bayston, M.Med.Sci., M.I.Biol., University Department of Paediatrics, Children's Hospital, Sheffield S10 2TH. 